\title{
Vom Bedürfnis zu glauben zum religiösen Grundakt
}

Eine „schwache“ Wesensanalyse des postsäkularen religiösen

Bewusstseins

Du besoin de croire à l'acte religieux fondamental. Une analyse eidétique " faible " de la conscience religieuse post-séculière

From the Need to Believe to the Basic Religious Act. A "Weak" Eidetic Analysis of Postsecular Religious Consciousness

\section{Peter Gaitsch}

\section{OpenEdition}

Journals

Édition électronique

URL : http://journals.openedition.org/ceg/2539

DOI : $10.4000 /$ ceg. 2539

ISSN : 2605-8359

Éditeur

Presses Universitaires de Provence

Édition imprimée

Date de publication : 26 avril 2018

Pagination : 25-35

ISBN : 979-10-320-0160-8

ISSN : 0751-4239

\section{Référence électronique}

Peter Gaitsch, « Vom Bedürfnis zu glauben zum religiösen Grundakt », Cahiers d'Études Germaniques [Online], 74 | 2018, Online erschienen am: 26 Oktober 2019, abgerufen am 10 Dezember 2020. URL http://journals.openedition.org/ceg/2539 ; DOI : https://doi.org/10.4000/ceg.2539 


\title{
Vom Bedürfnis zu glauben zum religiösen Grundakt
}

\author{
Eine „schwache“ Wesensanalyse des postsäkularen \\ religiösen Bewusstseins
}

\author{
Peter Gaitsch \\ Karl-Franzens-Universität Graz
}

Mein Beitrag ist dem Versuch gewidmet, Max Schelers religionsphänomenologischen Ansatz ${ }^{1}$, der Anfang der 1920er-Jahre für die katholische Religionsphilosophie einen Weg ,jenseits von Scholastik und Moderne“2 eröffnet hat, für eine Analyse der Religion im 21. Jahrhundert fruchtbar zu machen. Meine Überlegungen entfalten sich in vier Teilen. Der erste Teil präsentiert, unter dem Stichwort des „Postsäkularen“, eine Analyse der heutigen Situation der Religion und der Religionsphilosophie. Diese Analyse bildet die Grundlage für die Beurteilung zweier neuerer Zugänge, die ein vertieftes Verständnis der postsäkularen Religiosität versprechen: zum einen Julia Kristevas psychoanalytischen Zugang, zum anderen Bruno Latours soziologisch-existenziellen Zugang. Der abschließende vierte Teil antwortet auf die Probleme der beiden analysierten Zugänge, indem Schelers Ansatz in Stellung gebracht und gezeigt wird, inwieweit die von ihm skizzierte Wesensanalyse auch noch für die postsäkulare Religiosität einen angemessenen Verständnisrahmen bereitstellt.

\section{Das postsäkulare religiöse Bewusstsein und die "schwache" Religionsphilosophie}

Die postsäkulare Situation der Religion im 21. Jahrhundert bringt es mit sich, dass man sie legitim aus verschiedenen Gesichtspunkten beschreiben kann. Für die Religionsphilosophie erscheinen mir drei Gesichtspunkte von besonderer

1. Der zentrale Text ist: Max Scheler, „Probleme der Religion“, in Max Scheler, Vom Ewigen im Menschen, Bern / München, Francke, 1968 [1921], S. 101-354.

2. So lautet der treffende Titel eines Beitrags von Holger Zaborowski: Holger Zaborowski, „Jenseits von Scholastik und Moderne. Anmerkungen zu Max Schelers Phänomenologie der Religion“, Jahrbuch für Religionsphilosophie 2, 2003, S. 221-254. Vgl. zu dieser Charakterisierung von Schelers Religionsphilosophie bereits Richard Schaeffler, Die Wechselbeziehungen zwischen Philosophie und katholischer Theologie, Darmstadt, Wissenschaftliche Buchgesellschaft, 1980, S. 166. 
Relevanz. Wenn wir von einer Innen-Außen-Unterscheidung religiöser Partizipation ausgehen, kann man sie als den internen, den externen und den intermediären Gesichtspunkt bezeichnen.

(1) Der interne Gesichtspunkt ist der Gesichtspunkt des immer noch gläubigen Zeitgenossen, der darüber erstaunt, dass viele maßgebliche Zeitgenossen seinen Glauben nicht teilen und sogar für unvernünftig halten. (2) Der externe Gesichtspunkt ist der Gesichtspunkt des vollständig säkularisierten Zeitgenossen, der darüber erstaunt, dass Religion in einer Zeit der fortgeschrittenen wissenschaftlichen Naturbeherrschung immer noch als eine lebendige Kraft fortexistiert. (3) Der intermediäre Gesichtspunkt ist der Gesichtspunkt des, wie ich sagen möchte, genuin postsäkularen Zeitgenossen, der darüber erstaunt, dass er in sich selbst - nach der Auflösung jeder starken Glaubensüberzeugung immer noch eine Art religiöser „Attraktion“ antrifft.

Alle drei Gestalten von Staunen können den Anlass zu einer genuinen religionsphilosophischen Reflexion bilden: Die beiden ersten lassen sich als Varianten eines „starken“ Typs von Religionsphilosophie klassifizieren, da jeweils starke Überzeugungen, seien sie nun religiöser oder irreligiöser Natur, den Ausgangspunkt bilden. Die klassische philosophische Theologie und die nahezu ebenso klassische Religionskritik sind naheliegende Realisierungen dieses Typus. Gegenüber diesen beiden klassischen Gestalten der Religionsphilosophie möchte ich die dritte, genuin postsäkulare Gestalt des Staunens in den Fokus rücken, die Anlass zu einer „schwachen“ Religionsphilosophie gibt - „schwach“ im Sinne einer zögernden, unbestimmten, offenen, durch Attraktions- und Repulsionskräfte geprägten Überzeugung, von der ausgehend sich eine andere „Flugkurve“ religionsphilosophischer Überlegungen ankündigt. ${ }^{3}$ Mit dem Ausdruck „postsäkularer Standpunkt“ möchte ich hier also nicht primär eine soziologische Diagnose bezeichnen - das gesellschaftsrelevante Fortbestehen religiöser Gemeinschaften und das Fraglichwerden der undialektischen Säkularisierungsthese, die das Verhältnis von Wissenschaft und Religion als „Nullsummenspiel“ betrachtet ${ }^{4}$-, sondern vielmehr einen bestimmten Ort des

3. Die Idee eines religionsphilosophischen „schwachen Denkens“ nimmt zwar terminologische Anleihe bei Gianni Vattimo, die Konzeptionen sind aber wesensverschieden (vgl. Gianni Vattimo, Credere di credere, Milano, Garzanti, 1996; im Folgenden zitiert nach der deutschen Übersetzung: Gianni Vattimo, Glauben - Philosophieren. Aus dem Italienischen von Christiane Schultz, Stuttgart, Reclam, 1997). Während es Vattimo darum geht, den Prozess der Säkularisierung und eine Ontologie der Schwächung als den „kenotischen“ Sinn des christlichen Glaubens zu begreifen, geht es mir hier darum, den religiösen Grundakt zu begreifen, der noch diesseits einer starken Glaubensüberzeugung liegt.

4. Zu dieser Diskussion siehe vor allem Jürgen Habermas, „Glauben und Wissen. Friedenspreisrede 2001“, in Jürgen Habermas, Zeitdiagnosen. Zwölf Essays 1980 - 2001, Frankfurt / Main, Suhrkamp, 2003, S. 249-262, hier S. 251; Hans Joas, „Religion post-säkular? Zu einer Begriffsprägung von Jürgen Habermas“, in Hans Joas, Braucht der Mensch Religion? Über Erfahrungen der Selbsttranszendenz, Freiburg / Basel / Wien, Herder, 2004, S. 122-128. Habermas geht in seinem Beitrag aber über eine bloß soziologische Diagnose der Postsäkularität hinaus. Seine Konzeption einer postsäkularen Religionsphilosophie gleicht der vorliegenden darin, dass er die profane liberale Vernunft als autonome „dritte Partei zwischen Wissenschaft und Religion“ (Habermas, „Glauben und Wissen“, S. 251) ansetzt, die im Sinne einer positiven 
Denkens: eine interessierte (nicht-neutrale) mittlere Position, die „zwischen den Stühlen“ der Proponenten mit ihren jeweiligen „dichten Beschreibungen (thick descriptions)“ ${ }^{5}$ religiöser bzw. irreligiöser Erfahrung sitzt: auf der einen Seite dem Angehörigen einer religiösen Gemeinschaft mit seinem klar artikulierbaren Glaubensbekenntnis, auf der anderen Seite dem säkularen Religionsverächter mit seinem oft ebenso klar artikulierbaren szientifischen Weltbild.

Der postsakuläre Standpunkt zwischen den Stühlen dürfte gesamtgesellschaftlich keine Mehrheit repräsentieren, aber er ist auch nicht bloß das intellektuelle Phantasma von Religion. Wenn sich ausgehend von diesem Standpunkt eine „schwache“ Religionsphilosophie als ein eigener Typus ausbilden können soll (wenn die postsäkulare „schwache“ Überzeugung also tatsächlich einen Stand-Punkt und nicht bloß ein defizitäres Übergangsphänomen, ein „laues“ Dazwischen bezeichnet, das einfach nur durch die Wiedergewinnung einer starken Überzeugung überwunden werden muss), dann impliziert dies die Hypothese, dass es einen religiösen Grundakt des Menschen diesseits des (theologisch als „Gnade“ zu qualifızierenden) Glaubens gibt. Der Standpunkt der schwachen Religionsphilosophie, den ich für die folgenden Überlegungen einnehmen möchte, bewegt sich also in einer spezifischen In-Differenz, insofern aus seiner Sicht der Unterschied zwischen Glauben und Nichtglauben, aber auch der Unterschied zwischen einer Erfahrung von Seinsfülle und einer Erfahrung von Leere, d.h. der Unterschied zwischen westlichem und östlichem religiösen Bewusstsein ${ }^{6}$, für das postsäkulare religiöse Bewusstsein keinen Unterschied machen.

Im Folgenden werden die neueren Ansätze von Julia Kristeva und Bruno Latour zum Verständnis von Religion herangezogen, um zu ermitteln, was sie zur Analyse des postsäkularen religiösen Bewusstseins beitragen. Mit der „Bedürfnis“-Formel („Bedürfnis zu glauben“) stellt Kristeva einen Gedanken ins Zentrum, der die postsäkulare Situation der Religion unmittelbar zu erschließen scheint; und die oben angeführten Charakterisierungen der schwachen, zaudernden religiösen Überzeugung auf dem postsäkularen Standpunkt erinnern nicht zufällig an die Merkmale, die Latour von seinem persönlichen Verhältnis zur Religion zeichnet.

„rettenden“ Säkularisierung die „Übersetzung“ unabgegoltener Sinnressourcen religiöser Traditionen zu leisten hat. Habermas verweist außerdem auf die windungsreiche Geschichte der philosophischen Anverwandlung religiöser Gehalte von Kant bis Derrida, in der eine solche Konzeption zu situieren ist.

5. Terence Cuneo, Ritualized Faith: Essays on the Philosophy of Liturgy, Oxford, Oxford University Press, 2016, S. 15.

6. Vgl. die exemplarische Darstellung von Keiji Nishitani, Was ist Religion? Vom Verfassser autorisierte deutsche Übertragung von Dora Fischer-Barnicol, Frankfurt am Main, Insel, 1982. 


\section{Das „Bedürfnis zu glauben“ und die phänomenologische Grundlegung der Religionspsychologie}

Julia Kristevas Analyse des postsäkularen religiösen Bewusstseins besteht in einer kreativen Anwendung psychoanalytischer Begriffe und Theoriestücke auf religiöse Phänomene. Es geht ihr um eine Deutung der „psychischen Realität“7 der gläubigen Subjekte, die mit einem identifikatorischen Bezug auf spezifische religiöse Gehalte korreliert. Zwei herausragende Beispiele dafür sind einmal ihre Deutung der psychischen Realität, die mit dem identifikatorischen Verständnis der christlichen Passionsgeschichte gekoppelt ist, in dem sie einen das Leiden sublimierenden („durchquerenden“) Zug feststellt ${ }^{8}$, und ferner ihre Deutung der ekstatischen Mystik Teresa von Avilas, deren spezifische Religiosität Kristeva als ein Alternieren von entsexualisierender Idealisierung und Resexualisierung versteht. ${ }^{9}$ In derartigen ambivalenten psychischen Realitäten sieht Kristeva eine die Humanisierung und Säkularisierung anstoßende Psychodynamik am Werk, die ihrer Auffassung nach die jüdisch-christliche Tradition gegenüber dem Islam auszeichnet. ${ }^{10}$ Im Kern bietet sie jedoch eine psychoanalytische Ursprungsklärung des „Bedürfnisses zu glauben“ an. Auf diese möchte ich mich an dieser Stelle konzentrieren.

Das „Bedürfnis zu glauben“ wird von Kristeva als „vorreligiös“"11 bezeichnet, da es für das menschliche psychische Leben „konstitutiv“ " ${ }^{12}$ ist und jeder spezifisch religiösen Artikulationsform vorausliegt und daher auch noch dem postsäkularen Bewusstsein zugrunde liegt. Kristeva deutet den Glauben als „Idealitätskrankheit“ bzw. „Paradies-Syndrom“13 und beschreibt ihn idealtypisch als adoleszente Bewusstseinsformation: Die triebhafte Suche nach Befriedigung durch ein Objekt wird angesichts der enttäuschenden Realitätserfahrung idealisiert und verabsolutiert. Diese Verabsolutierung besteht im Glauben an die Existenz eines „absolut befriedigenden Anderen“, eines Objekts, das „absolute Erfüllung““ 14 verheißt. Ein entscheidender Zug des Bedürfnisses zu glauben liegt darin, dass es sich um ein „Amalgam“15 von Triebregung und Idealisierung handelt: „Der Schatten des Ideals ist auf den adoleszenten Trieb gefallen, um sich im Bedürfnis zu glauben zu kristallisieren“"16, wie es zusammenfassend heißt. Auf dieser Basis

7. Julia Kristeva, Bisogno di credere. Un punto di vista laico, Rom, Donzelli Editore, 2006 [italienische Originalausgabe], im Folgenden nach der deutschen Übersetzung zitiert: Julia Kristeva, Dieses unglaubliche Bedürfnis zu glauben. Aus dem Französischen von Eva zum Winkel unter Mitarbeit der Herausgeber. Hrsg. und mit einem Nachwort von Eberhard Th. Haas und Elisabeth Van Quekelberghe, Gießen, Psychosozial-Verlag, 2014, hier S. 21 u. 70.

8. Ibid., S. 72ff. u. $103 \mathrm{ff}$.

9. Ibid., S. 63.

10. Ibid., S. 80f. u. 97.

11. Ibid., S. 17.

12. Ibid., S. 27 u. 37.

13. Ibid., S. 32 bzw. 30.

14. Ibid., S. 31 u. 30.

15. Ibid., S. 32.

16. Ibid., S. $33 f$. 
kann sich und soll sich nach Kristevas Vorstellung in der weiteren psychischen Reifung ein humanisierender Sublimierungsprozess des Bedürfnisses zu glauben entfalten; dies setzt aber die Anerkennung des Bedürfnisses voraus. ${ }^{17}$

Diese genealogische Erklärung des religiösen Bewusstseins gerät nach meinem Dafürhalten in zwei Probleme, in ein thematisches und in ein methodisches. Das thematische Problem besteht darin, dass das religiöse Bewusstsein von einem Glaubensakt her definiert wird. Dies ist in der postsäkularen Situation aber keineswegs selbstverständlich. Diese Einsicht ist im übrigens bereits bei Kristeva angelegt, die neben dem religiösen Glauben auch noch einen „Zugang zum Heiligen“" kennt, was man landläufig gleichfalls als ein „religiöses“ Bewusstsein kennzeichnen würde. Es gibt also die Möglichkeit eines religiösen Bewusstseins, das sich gegenüber dem Unterschied zwischen Glauben und Nichtglauben indifferent verhält und dieses sollte uns im Sinne der schwachen Religionsphilosophie vor allem interessieren. Angesichts dessen gehen Kristevas übrige Analysen ins Leere, insofern sie sich auf den Glauben und seine Sublimierung fokussieren.

Das methodische Problem ist noch schwerwiegender und besteht darin, dass Kristevas Bestimmung des Glaubens bzw. des religiösen Grundaktes nicht in eine phänomenologische Sinnanalyse des Glaubens bzw. des religiösen Grundaktes eingebettet ist. Es wird der Eindruck erweckt, als ob das, was „religiöser Glaube“ heißt, entweder immer schon allseits bekannt ist und daher vorausgesetzt werden darf oder aber keiner immanenten Sinnerhellung zugänglich ist, wodurch eine eigentlich spekulative externe Sinnzuschreibung, von der Art „Glauben ist die Suche nach absoluter Befriedigung“, legitimiert wird. Dagegen ist einzuwenden, dass erstens der Sinn von „religiöser Glaube“ unklar ist und nach einer vorgängigen philosophischen Bedeutungsklärung verlangt und dass zweitens sich diese Bedeutungsklärung auf eine immanente Sinnanalyse des religiösen Aktes stützen kann und muss. Das bedeutet, dass auch eine psychoanalytische Erklärung sich mit dem vorgängig analysierten Phänomengehalt des Glaubens (bzw. des religiösen Grundaktes) auseinandersetzen und gute Gründe anführen muss, wenn sie dem manifesten Sinngehalt des Phänomens widerspricht. In diesem Sinne verlangt die Religionspsychologie nach einer phänomenologischen Grundlegung.

Dies ist die systematische Einsatzstelle von Schelers Analyse. Für den Augenblick genügt der Hinweis, dass Scheler in der Aktlehre eine "Noetik“ genannte Untersuchungsrichtung kennt, die der religionspsychologischen Untersuchung vorgelagert ist, da Erstere die Aufgabe hat, die „Sinnlogik“ des religiösen Aktes zu bestimmen. ${ }^{19} \mathrm{Um}$ den Unterschied zu verdeutlichen, nennt Scheler das Beispiel des Gebetsaktes: Die psychische Realität, die in einem Betenden vor sich geht, ist

17. Ibid., S. 27.

18. Ibid., S. 9; etwas obskur erläutert als die „Durchquerung des Undenkbaren durch das Denken“ (ibid. S. 108).

19. Vgl. Scheler, „Probleme der Religion”, S. $151 \mathrm{ff}$. 
für das Wesen des Gebetsaktes so gleichgültig, wie das Magendrücken oder die Phantasiebilder eines Mathematikers, der über ein Zahlproblem nachdenkt, für die Noetik des Zahldenkens ist. Der Gebetsakt ist nur vom Gebetssinn her zu bestimmen, und wie die psychischen Materialien psychologisch zusammengesetzt sind, die in diesem Akte verwendet oder verbraucht werden - wie sie sich etwa zusammensetzen aus Empfindungen, Gefühlen, Vorstellungen, Bedeutungsakten, Worten, Ausdrucksäußerungen, Handlungen -, das geht die religiöse Noetik überhaupt nichts an. ${ }^{20}$

Die noetische Bestimmung eines Aktes ist vorausgesetzt, um wissen zu können, um was für eine Art von Akt es sich überhaupt handelt. Da diese deskriptive Dimension der Analyse bei Kristeva aber völlig fehlt, ist ungesichert, ob die Bestimmungen, die bei ihr den impliziten Status von Wesensbestimmungen des Glaubens bzw. des religiösen Grundaktes einnehmen („Suche nach absoluter Befriedigung“"), nicht nur akzidentieller Natur sind. Genau das gilt es aber mittels einer noetischen Analyse vorab zu klären.

\section{Das Problem der Umgrenzung des postsäkularen religiösen Bewusstseins}

Anders als Kristeva gerät Bruno Latour, der in Jubilieren versucht, den Sinn religiöser Rede über die performative Dimension der Rede zwischen Liebenden zu explizieren, nicht in die bei Kristeva diagnostizierten beiden Probleme. Erstens ist sein Thema nicht der religiöse Glaube, sondern ein religiöser Bezug diesseits der Differenz von Glauben und Nicht-Glauben, eine existenzielle Attraktion gegenüber religiösen Dingen (Liturgie, Heilige Schrift, u.a.), die sich nicht in einem Glauben erfüllt. Das Fehlen von Glauben wird als religiös nicht-defizitärer, nicht überholungsbedürftiger Standpunkt erlebt, der nicht durch einen „Willen zum Glauben“, einen „Glauben an den Glauben“ ${ }^{21}$ oder ein „Bedürfnis zu glauben“ kompensiert werden muss. Denn Latour besetzt das Zögern und Zaudern mit einem religiösen Tiefensinn:

Ich kann diesem Zögern nicht entkommen, das nichts mit einem unsicheren Schwanken zwischen Unglauben und Glauben zu tun hat, zwischen „ich speie alles aus“ oder ,ich schlucke alles“. Das Zögern stellt die Sache selbst dar, ist immer neu aufzunehmen und neu $\mathrm{zu}$ formulieren. ${ }^{22}$

Sein Ausgangspunkt der Analyse entspricht somit genau dem eingangs erläuterten intermediären Standpunkt des genuin postsäkularen religiösen Bewusstseins.

Zweitens finden sich bei Latour Ansätze zu einer religiösen Noetik, sodass er dem methodischen Problem Kristevas von vornherein entgeht. Es ist Latours

20. Ibid., S. $151 \mathrm{f}$.

21. Bruno Latour, Jubiler - ou les tourmentes de la parole religieuse, Paris, Le Seuil, 2002, im Folgenden nach der deutschen Übersetzung zitiert: Bruno Latour, Jubilieren. Über religiöse Rede. Aus dem Französischen von Achim Russer, Berlin, Suhrkamp, 2016, hier S. 10 u. 60. Vgl. zum affirmativen Gebrauch dieser Formel: Vattimo, Glauben - Philosophieren, S. 76. Eine kritische Haltung zu dieser Formel findet sich bereits in Scheler, „Probleme der Religion“, S. 241.

22. Latour, Jubilieren, S. 215. 
ausdrückliches Ziel, die Sinnlogik des religiösen Grundakts auf dem Wege einer erstpersonalen Analyse zu klären. Da aber Latour kein Phänomenologe ist, erhält das Erstpersonale bei ihm sogleich den sehr persönlichen Charakter eines Ringens, der eigenen Erfahrung von zögerlicher religiöser Attraktion einen guten Sinn abzugewinnen. Zu diesem Zweck zieht Latour die Erfahrung des krisenhaften Gesprächs zwischen Liebenden heran, die ihm als Grundlage für die Explikation der religiösen Rede dient. In religiöser Rede geht es, wie im Gespräch der Liebenden (das sich rund um die Frage: „Liebst du mich?“ dreht), nicht um die Übertragung eines verborgenen Informationsgehalts („Geheimnis“) in der Art referenzieller Sprache („Zugang zu Fernem“), sondern performativ um eine „Logik der Transformation“23, nämlich um die Hervorbringung von Nähe und Gegenwart: „die sogenannt religiösen Worte haben keinerlei Referenz - nicht mehr als der Austausch zwischen Liebenden“" ${ }^{24}$. Wie bei den Liebenden geht es gemäß Latour auch bei den Religiösen um ein interpersonales Einandergegenwärtig-werden und um eine Nähe zueinander. ${ }^{25}$ Die Differenz zwischen liebender und religiöser Rede, für die das Pfıngstereignis Pate steht, besteht für Latour allein im Modus der Nähe und in der Bezugsweite der Nähe: Der religiöse Modus ist eine Erneuerung des Geistes (statt des Eros), und der religiöse Bezug ist im Unterschied zum Liebespaar nicht exklusiv, sondern konkret-universell. Letzteres bedeutet, dass es in der religiösen Nähe darum geht ein allumfassendes Volk zu werden: „ein Volk, das per definitionem nie mit irgendeiner sprachlichen, ethnischen oder kulturellen Grenze koinzidiert“26. Das ist Latours „virtueller Katholizismus eines neu zu erweckenden Volkes“ ${ }^{27}$. In ihm ist das Wort „Gott“ ein Synonym für das, „was Mitmenschen erzeugt“" ${ }^{28}$.

Doch auch wenn Latour der postsäkularen Situation des religiösen Bewusstseins auf diese Weise gerecht wird und außerdem eine Art von noetischer Analyse liefert, bleibt ein Problem bestehen, das Problem der Umgrenzung des religiösen Aktes. Dieses Problem manifestiert sich bei Latour in zwei Aspekten. Erstens beschreibt er den religiösen Grundakt nicht als einen Akt sui generis, sondern erläutert ihn über die zwischenmenschliche Liebesrede, sodass er als eine Hervorbringung von universeller zwischenmenschlicher Nähe gedeutet wird. Für Latour gibt es also keine genuin religiöse Noetik des religiösen Grundakts, was sich überdies an der Tendenz zur Funktionalisierung der religiösen Rede („Erzeugung von Nähe“) deutlich zeigt. Zweitens spricht Latour dem religiösen Grundakt jeglichen transzendenten Gegenstandsbezug ab. ${ }^{29}$ Phänomenologisch gesprochen ist seine noetische Analyse also nicht mit einer noematischen Analyse des religiösen Grundaktes verbunden. Da somit Latour dem religiösen Grundakt weder eine eigene Aktart noch eine eigene Gegenstandssphäre zuschreibt, lassen

\footnotetext{
23. Ibid., S. 142.

24. Ibid., S. 43.

25. Ibid., S. 167.

26. Ibid., S. 169.

27. Ibid., S. 235.

28. Ibid., S. 192.

29. Ibid., S. 237.
} 
sich in seinem Ansatz das Religiöse und das Nicht-Religiöse nicht wesenhaft voneinander abgrenzen und das Religiöse erhält den Charakter eines bloßen Anhängsels an andere Aktarten (hier: die Nähe hervorbringende Rede) und Gegenstandsbezüge (hier: den interpersonalen Bezug).

Die Frage bleibt, ob dies tatsächlich das letzte Wort bezüglich des religiösen Grundaktes sein kann und ob es nicht möglich ist, das postsäkulare religiöse Bewusstsein erfolgreich einer („schwachen“) Wesensanalyse zu unterziehen. Mit diesem Fokus wollen wir uns abschließend Schelers religionsphänomenologischem Ansatz zuwenden.

\section{Postsäkulare Wesenseinsichten in den religiösen Grundakt}

Scheler kommt in seiner wesensphänomenologischen Analyse des religiösen Grundakts zum Ergebnis, dass sich der religiöse Grundakt gegenüber weltlichen Akten durch einen spezifischen Gegenstandsbezug, mit dem eine spezifische Gegebenheitsweise einhergeht, auszeichnet. Der Gegenstand, genannt das „Göttliche“, ist von der Art, dass er sich selbst mitteilt, dass er sich im naturhaften Sein ausdrückt. Scheler bezeichnet die damit korrelierende Gegebenheitsweise als „natürliche Offenbarung“. Das Göttliche offenbart sich im naturhaften Sein. Diese natürliche Offenbarung, die für Scheler neben dem metaphysischschlussfolgernden Denken und der partikularen-positiven Offenbarung ein „drittes Erkenntnisprinzip“30 innerhalb des religiösen Gebiets bildet, umreißt das spezifische Themenfeld der phänomenologischen Religionsphilosophie. Sie hat also die religiösen Erkenntnisse, die der natürlichen Offenbarung entstammen, $\mathrm{zu}$ formulieren.

In Schelers Entwurf von 1921 führt die Auswertung der natürlichen Offenbarung $\mathrm{zu}$ einer klassischen theistischen Position, die sich vom scholastischen Denken hauptsächlich dadurch unterscheidet, dass sie diese Position nicht durch metaphysisch-schlussfolgerndes Denken, sondern durch genuine Evidenzeinsichten $\mathrm{zu}$ erreichen beansprucht. Ich möchte die damit verbundene Problematik an dieser Stelle jedoch ausklammern, da es mir hier allein darum geht zu untersuchen, welche von Schelers beanspruchten Wesenseinsichten sich noch positiv auf das postsäkulare religiöse Bewusstsein, das sich nicht auf einen theistischen Gottesglauben festlegen lässt, beziehen lassen. Was sind also Schelers bleibend gültige Resultate einer „schwachen“ Wesensanalyse?

Auf die postsäkulare Situation anwendbar ist erstens die mit dem Ausdruck „natürliche Offenbarung“ bezeichnete Annahme eines spezifischen Gegenstandsbezugs des religiösen Grundakts, unter der Voraussetzung allerdings, dass der Offenbarungsakt seitens des Göttlichen nicht sofort als personaler Akt

30. Max Scheler, „Vorrede zur zweiten Auflage“ [1922], in Max Scheler, Vom Ewigen im Menschen, Bern / München, Francke, 1968, S. 21. 
gedeutet wird, wie das bei Scheler geschieht ${ }^{31}$. Denn nicht jede offenbarende Selbstdarstellung muss zwangsläufig als personaler Vollzug gedeutet werden. Das hier implizite Entweder-Oder von Naturalismus und Theismus ${ }^{32}$ ist allzu simplifizierend, wie offenbar Scheler selbst erkannt hat, als er sich nach 1922 (panentheistischen) Zwischenpositionen zuwandte und Gott auf eine nicht mehr personalistische Weise als einen durch den geschichtlichen Weltprozess „werdenden Gott“" konzipierte. ${ }^{33}$

„Natürliche Offenbarung“ bedeutet aber grundsätzlich, dass das Göttliche die Ursache für den religiösen Grundakt des Menschen bildet, indem es zugleich eine eigene Sphäre der Wirklichkeit - die „Absolutsphäre“, wie Scheler sagt eröffnet, der sich kein Mensch entziehen kann:

Nur ein reales Seiendes mit dem Wesenscharakter des Göttlichen kann die Ursache der religiösen Anlage des Menschen sein, d.h. der Anlage zum wirklichen Vollzug jener Aktklasse, deren Akte durch endliche Erfahrung unerfüllbar sind und gleichwohl Erfüllung fordern. ${ }^{34}$

Daraus folgt zweierlei: Erstens wird durch die natürliche Offenbarung des Göttlichen der religiöse Grundakt zu einem notwendigen Vollzug des Menschen, denn die einmal geoffenbarte Absolutsphäre der Wirklichkeit kann nicht nicht besetzt werden. Es gibt lediglich eine Wahl zwischen „Gott oder Götze“35: Entweder wird die Absolutsphäre mit dem ihr entsprechenden Göttlichen (wieder-)besetzt oder sie wird durch einen verabsolutierten endlichen Gehalt, der aus einer anderen Wirklichkeitssphäre stammt, neu besetzt. Zweitens ergibt sich aus der natürlichen Offenbarung ein negatives Kriterium zur Unterscheidung zwischen echter und vermeintlicher Offenbarung: Jeder vermeintliche Offenbarungsgehalt, der sich auf ein weltliches Wissen zurückführen lässt, ist kein echter Gehalt der natürlichen Offenbarung. Das heißt aber positiv gewendet, dass das „fortschreitende Weltwissen“ als „Reduktionsmittel aller bloß vermeinten Offenbarung auf ihren ,reinen“ Gehalt“36 dienen kann. Dieser Hinweis ist doppelt bedeutsam: einmal dadurch, dass er zeigt, dass der Rekurs auf religiöse Evidenzen nicht zur irrationalistischen Kritiklosigkeit gegenüber dem Gegebenen verdammt; und zusätzlich dadurch, dass der unabschließbare Fortschrittscharakter weltlichen Wissens ein selbstgewisses Ankommen bei der „reinen“ Offenbarung verunmöglicht. Der reine Gehalt der natürlichen Offenbarung bleibt somit ein regulatives Ideal, dass sich in keiner endgültigen Bestimmung erfüllen kann.

31. Vgl. Scheler, „Probleme der Religion“, S. 248.

32. Vgl. zum Beispiel die von Holm Tetens vorgelegte Skizze zu einer zeitgenössischen rationalen Theologie, die mit dieser Prämisse operiert: Holm Tetens, Gott denken. Ein Versuch über rationale Theologie, Stuttgart, Reclam, 2015.

33. Vgl. u.a. Max Scheler, „Philosophische Weltanschauung“ [1928], in Max Scheler, Späte Schriften, Bern / München, Francke, 1979, S. 75-84, bes. S. 83.

34. Scheler, „Probleme der Religion“, S. 255.

35. Ibid., S. 261.

36. Ibid., S. 352. 
Diese bleibende Unbestimmbarkeit zeigt sich auf noematischer Seite, also auf Seite der Wesenscharakterisierung des Göttlichen, darin, dass das natürlich geoffenbarte Göttliche nur mit „formalen Attributen“ bezeichnet werden kann, die die Frage der Geistigkeit und der Personalität des Göttlichen unentschieden lassen. Denn gemäß Scheler wird das Göttliche in der primären Erfahrung des religiösen Grundakts als das erfahren, was aus sich existiert (ens a se), was allwirksam und was in einem höchsten Sinne wertvoll, also heilig ist (summum bonum). Diese drei Merkmale sind „die einzigen, die das Gegenstandsgebiet einer religiösen Bewußtseinsweise unbedingt konstituieren und umgrenzen“" ${ }^{37}$. Man kann hinzusetzen, dass diese Bestimmung auch noch für das postsäkulare religiöse Bewusstsein gilt und dass das postsäkulare Zögern erst dort einsetzt, wo es um eine (theistische oder nicht-theistische) Fortbestimmung dieser formalen Attribute geht.

Auf noetischer Seite, also auf Seite der Wesenscharakterisierung des religiösen Grundakts, zeigt sich die bleibende Unbestimmtheit darin, dass wir nach Scheler nur „diagnostische“ Merkmale für das Vorliegen eines echten, d.h. irreduziblen religiösen Aktes haben. Scheler nennt drei solche Merkmale, die als Kriterien dienen können ${ }^{38}$ :

Ein religiöser Grundakt ist erstens welttranszendierend, d.h. er enthält eine Vorstellung von Ganzheit, die sodann den Abstoßungspunkt bildet - der religiöse Grundakt zielt somit auf etwas, was im Ganzen nicht vorkommt.

Ein religiöser Grundakt ist auf dieser Basis zweitens empirisch unerfüllbar (,empirisch grundlos wie ziellos“39), und zwar evident unerfüllbar. Das ist das negative Kriterium, das aus der Welttranszendierung folgt: Ein religiöser Grundakt muss so verfasst sein, dass seine Erfüllung weltlich gesehen unmöglich ist. Kein vorstellbarer endlicher Gehalt darf ihn erfüllen können; andernfalls würde sich zeigen, dass er nur auf einem endlichen Mangel, nicht auf einem welttranszendierenden Überschuss, beruht. Scheler illustriert diesen Sachverhalt unter anderem am Beispiel des genuin religiösen „Liebens“ und am Beispiel des genuin religiösen „Hoffens“:

[W]ir finden uns gerichtet auf etwas, an dessen Stelle kein endliches, wie immer liebenswürdiges Gut treten kann, da die religiöse Liebe die Wesensartung solcher Güter transzendiert. [...] Im „religiösen“ Hoffen hoffen wir etwas, was wir nie erfahren haben und von dem wir wissen, daß wir es nie haben erfahren können. ${ }^{40}$

Da es somit im weltlichen Bereich keine funktionalen Äquivalente für echte religiöse Akte geben kann, stößt eine funktionalistische Betrachtung an dieser Stelle an ihre Grenze. ${ }^{41}$

37. Ibid., S. 169.

38. Ibid., S. 245-249.

39. Ibid., S. 247.

40. Ibid., S. 246.

41. Vgl. die ähnliche Überlegung von Hans-Joachim Höhn, Zeit und Sinn. Religionsphilosophie postsäkular, Paderborn u.a., Schöningh, 2010, S. 154. 
Ein religiöser Grundakt hat drittens einen rezeptiven und responsiven Grundzug. Das ist die positive Kehrseite zur weltlichen Unerfüllbarkeit, die der natürlichen Offenbarung entspricht. In den Worten Schelers:

Der religiöse Akt fordert - im Unterschiede zu allen sonstigen Erkenntnisakten, auch denen der Metaphysik - eine Antwort, einen Wider- und Gegenakt seitens eben des Gegenstandes, auf den er seinem intentionalen Wesen nach abzielt. ${ }^{42}$

Genauer müsste man allerdings sagen, dass primär nicht der Gegenstand als antwortend erfahren wird, sondern dass sich das religiöse Bewusstsein selbst als antwortend verstehen muss, insofern ihm nämlich die natürliche Offenbarung immer schon zuvorkommt.

Die drei angeführten Merkmale sind Kriterien des religiösen Grundakts, die auch noch für das postsäkulare religiöse Bewusstsein gelten, da sie die bleibende Unbestimmbarkeit des Göttlichen nicht verletzen.

Resümierend können wir als das wichtigste Resultat einer an Scheler angelehnten „schwachen“ Wesensanalyse festhalten, dass sich das postsäkulare religiöse Bewusstsein nicht (wie bei Latour) als ein Anhängsel ursprünglicherer Bewusstseinsakte verstehen muss: dies in erster Linie deswegen, da es seine eigene Gegenstandssphäre („Absolutsphäre“) hat. Bis auf wenige formale Attribute und diagnostische Kriterien sieht sich das postsäkulare Bewusstsein aber nicht in der Lage, die Absolutsphäre näher zu bestimmen. Die natürliche Offenbarung erscheint als eine Gegebenheit ohne bestimmbare Botschaft. Vor diesem Hintergrund können Versuche wie derjenige Latours wieder in ihr Recht gesetzt werden..$^{43}$ Sie sind erfinderische Versuche, dem Unbestimmbaren des Absoluten eine im höchsten Sinn existenziell bedeutsame Stelle (als normative Quelle) im postsäkularen Leben zu geben. Vor diesem Hintergrund kann außerdem der „Bedürfnis“-Formel ein von Kristevas psychoanalytischer Analyse abweichender Sinn zugeschrieben werden: Wenn es ein echtes, irreduzibles religiöses Bedürfnis gibt, dann meldet sich darin nicht ein humanes Defizit, das sich in der religiösen Vorstellungswelt funktional überkompensiert und nach einer Re-Humanisierung durch sublimierenden Abbau der religiösen Idealisierung verlangt, wie es Kristeva nahelegt - sondern vielmehr ein nicht funktionalistisch abzugeltender humaner Überschuss, bei dem es nur gilt, ihn sich als absoluten Quellgrund in seiner bleibenden Unbestimmbarkeit möglichst rein zu bewahren.

42. Scheler, „Probleme der Religion“, S. 248.

43. $\mathrm{Zu}$ denken ist hier aber auch an andere neuere Arbeiten, die die Leerstelle bzw. die radikale Fremdheit als Quell- und Referenzpunkt einer authentischen religiösen Rede in den Mittelpunkt rücken: Alain Badiou, Saint Paul - La fondation de l'universalisme, Paris, PUF, 1997; Bernhard Waldenfels, „Religiöse Transzendenz“, in Bernhard Waldenfels, Hyperphänomene. Modi hyperbolischer Erfahrung, Berlin, Suhrkamp, 2012, S. 354-412. 
